Psychopharmacology, 2014. 231(7), 1351-1364

Trujillo-Pisanty et al.

3D analysis of DA receptor blockade

\title{
A new view of the effect of dopamine receptor antagonism on operant performance for
}

rewarding brain stimulation in the rat

I.Trujillo-Pisanty, K. Conover \& P. Shizgal*

Center for Studies in Behavioural Neurobiology /

Groupe de Recherche en Neurobiologie Comportementale

Concordia University

7141 Sherbrooke Street West, SP-244

Montréal, Québec, H4B 1R6, Canada

* Communicating author: e-mail: peter.shizgal@,concordia.ca, telephone: +1 (514) 848-2424 ext 2191, fax: +1 (514) 848-2817

Acknowledgements: The research was supported by a grant to PS from the Canadian Institutes of Health Research (\#MOP-74577), a group grant from the "Fonds recherche Québec - santé" to the "Groupe de Recherche en Neurobiologie Comportementale" / Center for Studies in Behavioural Neurobiology (Shimon Amir, p.i.), support for PS from the Concordia University Research Chairs program, and scholarships to ITP from the "Consejo Nacional de Ciencia y

Tecnologia" (CONACYT, \#209314) and "le Ministère de l'Éducation, du Loisir et du Sport du Québec" (PBEEE-1M, \#140498). David Munro built and maintained the computer-controlled equipment for experimental control and data acquisition. Software for experimental control and data acquisition was written and maintained by Steve Cabilio. The authors thank Brian Dunn for helpful comments on the manuscript. 


\section{Abstract}

Rationale. Previous studies of neuroleptic challenges to intracranial self-stimulation (ICSS) employed two-dimensional (2D) measurements (curve shifts). Results so obtained are ambiguous with regard to the stage of neural processing at which the drug produces its performance-altering effect. We substituted a three-dimensional (3D) method that measures reward-seeking as a function of both the strength and cost of reward. This method reveals whether changes in reward seeking are due to drug action prior to the output of the circuitry that performs spatiotemporal integration of the stimulation-induced neural activity.

Objectives. The aim of this study was to obtain new information about the stage of neural processing at which Pimozide acts to alter pursuit of brain stimulation reward (BSR).

Methods. Following treatment with Pimozide $(0.1 \mathrm{mg} / \mathrm{kg})$ or its vehicle, the proportion of trial time allocated to working for BSR was measured as a function of pulse frequency and opportunity cost. A surface defined by Shizgal's reward-mountain model was fitted to the drug and vehicle data.

Results. Pimozide lowered the cost required to decrease performance for a maximal BSR to half its maximal level but did not alter the pulse-frequency required to produce a reward of half-maximal intensity.

Conclusions. Like indirect dopamine agonists, Pimozide does not alter the sensitivity of brain reward circuity but changes reward-system gain, subjective effort costs, and/or the value of activities that compete with ICSS. The $3 \mathrm{D}$ method is more sensitive and informative than the $2 \mathrm{D}$ methods employed previously.

\section{Keywords}

1. Pimozide

2. opportunity cost

3. neuroeconomics

4. reward mountain

5. intracranial self-stimulation

6. ICSS

7. neuroleptics 


\section{Introduction}

Animals will work vigorously at operant conditioning tasks to receive electrical stimulation of the medial forebrain bundle (MFB) (Olds \& Milner 1954). This behavior, known as intracranial self stimulation (ICSS), has been used extensively to study the pharmacological basis of reward. In this paper, we extend prior work on the role of dopamine (DA) in ICSS by applying a new measurement method (Arvanitogiannis \& Shizgal 2008; Hernandez et al. 2010; Breton et al. 2013). The results reported here complement recent findings that cast the role of DA neurons in a new light (Hernandez et al. 2010; Hernandez et al. 2012).

The most widely adopted method for measuring ICSS, the curve-shift method, (Edmonds \& Gallistel 1974; Edmonds \& Gallistel 1977; Miliaressis et al. 1986), employs electrical stimuli of different strength (e.g., pulse-frequency or current) as the independent variable and a measure of response vigor (e.g., response rate) as the dependent variable. The stimulation strength that produces a criterial level of performance is determined under control and experimental conditions. Commonly, half-maximal performance serves as the behavioral criterion. The pulse frequency required to meet this criterion is designated $F_{m 50}$; this value specifies the position of the mid-point of the response rate-versus-pulse-frequency curve along the pulse-frequency axis. A decrease in $F_{m 50}$ corresponds to a leftward shift of the curve and is interpreted as facilitation of the rewarding effect, whereas an increase in $F_{m 50}$ corresponds to a rightward shift and is interpreted as attenuation of the rewarding effect. Dopaminergic agonists decrease $F_{m 50}$, whereas dopaminergic antagonists increase $F_{m 50}$ (Wise 1996). These findings constitute the principal foundation for a highly influential hypothesis: that the rewarding effects of electrical brain stimulation is encoded in the firing of dopamine (DA)-containing neurons.

Operant performance depends not only on reward strength but also on effort and opportunity costs (Niv et al. 2007; Breton et al. 2009; Hernandez et al. 2010), as well as on the value of activities, such as grooming, resting, and exploring, that compete with pursuit of the reward offered by the experimenter (Herrnstein 1970; Heyman \& Beer 1987; Petry \& Heyman 1997). Taking these variables into account challenges the assumption that changes in $F_{m 50}$ necessarily reflect facilitation or attenuation of the rewarding effect and offers alternate ways to interpret the effects of drugs that alter DA neurotransmission (Hernandez et al. 2010; Trujillo-Pisanty et al. 2011; Hernandez et al. 2012) .

Figure 1 integrates the processing of the stimulation-induced volley of action potentials that gives rise to brain stimulation reward (BSR) with task and environmental variables. The intensity of the rewarding effect is determined by spatial and temporal summation in a neural circuit or population that is activated trans-synaptically by the directly activated neurons. This circuit/ population has been dubbed the integrator. The behavior of the integrator is well described by a counter model (Gallistel et al. 1981; Simmons \& Gallistel 1994; Gallistel \& Leon 1991; Gallistel et al. 1991) that expresses the rewarding effect as a function of the aggregate rate of firing induced by the pulse train in the directly activated neurons. The rewarding effect grows non-linearly as a function of the induced rate of firing (Leon \& Gallistel 1992; Gallistel \& Leon 1991; Simmons \& Gallistel 1994) and the train duration (Sonnenschein et al. 2003). Later stages of neural processing compute a payoff by combining the peak reward intensity produced by the stimulation train with 
information about task variables, such as effort and opportunity costs (Arvanitogiannis \& Shizgal 2008; Hernandez et al. 2010; Shizgal 2012). In concert with environmental variables, such as alternate sources of reward, the payoff from BSR determines the proclivity of the animal to invest time and effort to obtain electrical stimulation.

The depiction in Figure 1 reveals an ambiguity inherent in the curve-shift method: $F_{m 50}$ values can be altered similarly by very different manipulations acting at different points in the neural circuitry that processes information about the reward, the operant task, and the test environment (Arvanitogiannis \& Shizgal 2008; Hernandez et al. 2010; Breton et al. 2013). For example, the effect of changing the current, a manipulation that alters the number of neurons providing input to the integrator, can be indistinguishable from the effect of manipulations that act beyond the output of the integrator, such as altering the opportunity cost of the reward (the time required to earn a pulse train) or the value of competing activities (Video 1). This ambiguity limits the inferences about the reward substrate that can be drawn from experiments in which ICSS is challenged by interventions such as drug administration, lesions, and alterations in physiological state.

By manipulating two independent variables rather than one, information lost in the curve-shift method can be retrieved, and the locus of action of an experimental manipulation can be resolved with respect to the output of the integrator. Instead of tracking shifts of a two-dimensional (2D) curve, shifts in the position of a three-dimensional (3D) surface are measured. Rats are trained to hold down a lever in order to receive rewarding stimulation. Both the pulse frequency and the cumulative time required to obtain a reward (the opportunity cost or price of the stimulation train) are varied across trials. The proportion of trial time invested in harvesting the reward (time allocation: TA) serves as the dependent variable. The resulting 3D representation, termed a reward mountain, expresses TA as a function of pulse frequency and price (Video 1).

In the $2 \mathrm{D}$ curve-shift method, $F_{m 50}$ locates rate-frequency curves along the axis representing the single independent variable. In the $3 \mathrm{D}$ method, a pair of location parameters play an analogous role by locating the TA surface in the space defined by the two independent variables. The stimulation frequency that produces half-maximal reward $\left(F_{h m}\right)$ determines the position of the surface along the pulse-frequency axis, whereas the price that produces half maximal TA for a maximal reward $\left(P_{e}\right)$ determines the position of the surface along the price axis. These location parameters reflect different stages of neural processing. Manipulations acting prior to the output of the integrator (e.g., changes in stimulation current or train duration) shift the 3D structure along the pulse-frequency axis, thus altering $F_{h m}$. Changes at or downstream from the output of integrator displace the three-dimensional structure along the price axis, thus altering $P_{e}$ (Figure 1, Video 1) (Arvanitogiannis \& Shizgal 2008; Hernandez et al. 2010; Breton et al. 2013).

The procedure based on the 3D reward-mountain model has been used successfully to disambiguate the level of neural processing at which DA agents act to alter ICSS of the MFB. The work shows that enhancement of DA signaling consistently increases the value of $P_{e}$ but rarely affects $F_{h m}$ (Hernandez et al. 2010; Hernandez et al. 2012). Based on the 2D curve-shift analysis, the effects of DA agents have been likened to those produced by changing the current (Edmonds \& Gallistel 1977; Miliaressis et al. 1986), a manipulation that acts upstream from the integrator. In contrast, the 3D analysis positions the locus of the drug effect at or beyond the output of the 
integrator, a stage at which multiple variables, including subjective effort costs, act to alter reward seeking (Figure 1; (Arvanitogiannis \& Shizgal 2008; Hernandez et al. 2010; Breton et al. 2013).

In the present study, we used the 3D method to assess the effects of Pimozide, a DA antagonist, on ICSS, thus complementing earlier work carried out with indirect DA agonists (Hernandez et al. 2010; Hernandez et al. 2012). On the basis of the prior work, we predicted that Pimozide would decrease $P_{e}$ without affecting $F_{h m}$.

\section{Materials and Methods}

Subjects. Eight, 350-400 g, male, Long-Evans rats (Charles River breeding farms) were housed in pairs in Plexiglas cages in a vivarium with a reversed $12 \mathrm{~h}$ dark/light cycle (lights "on" at 8:00 A.M.). All behavioral tests were conducted during the dark cycle (between 8:00 A.M. and 2:30 P.M.). Rats were housed individually following electrode implantation.

Electrode implantation. Atropine sulfate $(0.05 \mathrm{mg} / \mathrm{kg}$, s.c. $)$ was administered to reduce bronchial secretions, a ketamine-xylazine mixture (10/100 mg/kg, i.p.) was used to induce anesthesia, and penicillin $(0.3 \mathrm{ml} / \mathrm{kg}$, s.c.) was used to prevent infections. Xylocaine jelly was applied to the external auditory meatus to reduce discomfort from the stereotaxic ear bars. Once the rat was mounted on the stereotaxic frame, anesthesia was maintained by isoflurane vapor administered through a snout mask.

Six stainless-steel screws were threaded into pilot holes drilled in the skull. One end of a copper wire was wrapped around two of the screws, which served as the current return (anode); the other end was terminated in a gold-plated Amphenol connector. Monopolar stainless-steel electrodes were custom-made from 000 insect pins, which were insulated with Formvar enamel to within $0.5 \mathrm{~mm}$ from the bare tip; the unsharpened end was soldered to a copper wire, which, in turn, was attached to a gold-plated Amphenol connector.

The electrodes were aimed bilaterally at the MFB, at the level of the lateral hypothalamus (LH, AP: -2.8 from bregma, ML: \pm 1.7 , DV: 8.8-8.9 from skull surface), and secured to the skull with dental acrylic. The Amphenol connectors were inserted into a McIntyre miniature connector (Scientific Technology Centre, Carleton University, Ottawa, ON, Canada), which was attached to the skull and skull-screw anchors with dental acrylic. Rats were allowed at least five days of postsurgical recovery before behavioral screening commenced.

Apparatus. The experimental chambers $(30$ X $21 \mathrm{X} 51 \mathrm{~cm})$ have mesh floors and a transparent Plexiglas front panel. An amber flashing light is mounted $10 \mathrm{~cm}$ above the mesh floor, a retractable lever (ENV-112B, MED Associates) is mounted on the right side panel, and a $1 \mathrm{~cm}$ cue light is located $2 \mathrm{~cm}$ above the lever. An electrical swivel located at the top of the box allowed the animal to circle without tangling the stimulation leads.

A computer-controlled digital pulse generator and a constant-current amplifier were used to set the temporal parameters of the electrical stimulation and the pulse amplitude, respectively. A custom-written computer program ("PREF", Steve Cabilio, Concordia University, Montreal, QC, Canada) controlled the experiments and logged the data. 
Self-stimulation training. Each rat was screened to determine the electrode and the combination of electrical current and pulse frequency that best supported vigorous lever pressing with minimal motoric and/or aversive side effects. From this point onwards, the most effective electrode was used and the current was kept constant. Rats were trained to depress the lever for $4 \mathrm{~s}$ on a cumulative-handling-time schedule (Breton et al. 2009) to receive a $0.5 \mathrm{~s}$ train of $0.1 \mathrm{~ms}$ cathodal pulses. This schedule delivers a reward when the cumulative time that the lever has been depressed reaches an experimenter-defined criterion, called the price of the stimulation. Once performance on the cumulative handling-time schedule was stable, the rats were gradually trained on the different subtasks that provide the data required to fit the mountain model: frequency sweeps, price sweeps, and radial sweeps.

A frequency sweep consisted of eleven $80 \mathrm{~s}$ trials; given the $4 \mathrm{~s}$ price, the rat could harvest a maximum of 20 reinforcements per trial. Within each trial, the pulse-frequency and price were held constant. A black-out delay (BOD) followed delivery of the reward: during this delay, the lever was disarmed and retracted for $2 \mathrm{~s}$, and the trial-duration timer was paused. The pulse frequency of the stimulation on the first three trials of each sweep was set to the maximal value the rat could tolerate without manifesting pronounced forced movements or signs of aversion; the pulse frequency was then decreased in equal proportional steps across the remaining trials. The step size was adjusted for each rat so as to yield a sigmoidal relationship between TA and the logarithm of the pulse frequency, with an upper asymptote above 0.8 , a lower asymptote below 0.2 , and a transitional region positioned roughly mid-way between the upper and lower TA asymptotes. The first two trials of each sweep served as warm-ups and were excluded from the analysis. A $10 \mathrm{~s}$ inter-trial interval (ITI) signaled the beginning of a new trial; the amber light flashed during this period, and a non-contingent (priming) stimulation train at the maximal pulse-frequency was delivered at the $8 \mathrm{~s}$ and $10 \mathrm{~s}$ timepoints. The computer time-stamped and logged each transition of the lever (from up to down or down to up).

Once consistent sigmoidal-shaped TA-frequency curves were obtained, the rat was trained to perform on price sweeps. These consisted of eleven trials during which the reward strength was maximal, but the price was increased systematically across trials. The price on the first three trials was set to either 3 or $4 \mathrm{~s}$; the first two trials were considered warm-ups and were excluded form analysis. The trial duration was adjusted to allow the rat to harvest a maximum of twenty rewards per trial. The values of the ITI, BOD and priming parameters were the same as those used on frequency sweeps. During this phase of training, test sessions consisted of 3 price sweeps randomly intercalated with 4 frequency sweeps. The size of the proportional price changes was adjusted for each rat so as to produce well-formed sigmoidal TA-price curves, as described above in the case of the frequency sweeps.

Radial sweeps were incorporated in the training sessions once consistent sigmoidal TA-prices curves were obtained. On radial sweeps, we both decreased the pulse-frequency and increased the price systematically across trials. Eleven trials were run, with the first two defined as warm-ups and excluded from the analysis. The sizes of the proportional changes in pulse-frequency and price were adjusted so that the resulting TA-frequency and TA-price curves were sigmoidal and so that the trajectory of the radial sweep in the space defined by the pulse frequency and price (see the green points in Figure 5) passed close to the point defined by the two location parameters 
$\left(P e, F_{h m}\right)$. The empirical objective of the 3D analysis is to measure drug-induced shifts of this point. Thus it is important that behavioral measurements be obtained as close to the location parameters $\left(P e, F_{h m}\right)$ as possible. To achieve this, we used a simulator implemented by Yannick Breton in MATLAB (the MathWorks, Natick, MA) to aim the radial sweep at $\left(P e, F_{h m}\right)$ on the basis of the data from the frequency and price sweeps and estimates of slope parameters based on previous studies. As data obtained from radial sweeps accumulated, preliminary fits of the 3D reward-mountain model were performed as described below (Model fitting and comparisons), and the trajectory of the radial sweep was adjusted accordingly.

After the introduction of radial sweeps, test sessions consisted of 3 frequency sweeps, 2 price sweeps and 2 radial sweeps. The first sweep was always a warm-up frequency sweep, which was excluded from the analysis. We refer to the dataset composed of a frequency sweep, a price sweep and a radial sweep as a survey, the minimal dataset that suffices to fit the mountain model. Within a survey, sweep types were ordered pseudorandomly. Each test session included two surveys, performed sequentially. Four of these sessions were run, and then the data were fit using a resampling strategy, as described below (Model fitting and comparisons). If any of the sweeps lacked well-defined lower and upper asymptotes, or if the radial sweep strayed too far from $\left(P_{e}, F_{h m}\right)$, the pulse frequencies and/or prices were adjusted, and four extra sessions were run. This process was repeated until the data met the criteria, at which point the rats were considered ready for drug testing. Two rats failed to perform all of the stages of training described above and were excluded from the experiment.

Drug testing. Each drug and vehicle session started with a warm-up frequency sweep, followed by two surveys, which each required 2-3 h to complete. Each type of sweep within a survey was presented in pseudorandom order. Pimozide $(0.1 \mathrm{mg} / \mathrm{kg}$; Tocris Bioscience) was diluted in physiological saline and administered at a volume of $1.0 \mathrm{ml} / \mathrm{kg}$, i.p., $180 \mathrm{~min}$ before each behavioral test. This dose was determined from previous experiments (Atalay \& Wise 1983; Gallistel \& Freyd 1987) and from our own pilot tests, in which responding ceased or was unacceptably weak at doses of $0.25-1.0 \mathrm{mg} / \mathrm{kg}$ (data not shown). Rats were tested following vehicle injections on Mondays and Thursdays, and after drug injections on Tuesdays and Fridays. Wednesdays and weekends served as drug elimination days. During vehicle sessions, the rats were presented with the same sets of sweeps determined during training. On drug sessions, all prices from the price sweep were decreased by 0.1-0.2 $\log 10$ units (1.25-1.58 s) because our pilot tests suggested leftward shifts of this magnitude along the price axis. Had we not done this, we would have risked losing the upper asymptote of the price sweep, which would have compromised the fit of the model.

Six sessions, each 5-6 h long, were run with each rat in the vehicle and drug conditions, and thus, all rats received the $0.1 \mathrm{mg} / \mathrm{kg}$ dose of Pimozide six times. Rats Pharm 2 and Pharm 3 also received four injections each at Pimozide doses ranging from $0.25-1.0 \mathrm{mg} / \mathrm{kg}$ during pilot tests carried out prior to the experiment proper.

A second frequency sweep, carried out at a lower price, had to be included in all drug sessions conducted with subject Pharm3. This was necessary because in this subject, TA decreased very steeply as a function of price (data not shown). Performance on the $4 \mathrm{~s}$ frequency sweep no longer 
attained a sufficiently high TA to provide a good estimate of the upper asymptote. The addition of the low-price frequency sweep provided the needed information. The objective of estimating the upper asymptote in the drug condition was addressed in rats Pharm10, Pharm11 and Pharm13 by decreasing the price during frequency-sweep trials to $3 \mathrm{~s}$.

On average, it took three months following surgery to finish the experiment with each rat.

Following data acquisition, the rats were overdosed with ketamine-xylazine. The location of the electrode tip was determined by staining the tissue with the Prussian-Blue method and examination of brain sections by means of low-magnification microscopy (Hernandez et al. 2006).

Model fitting and comparisons. The mountain model and the fitting approach have been described in detail elsewhere (Hernandez et al. 2010; Trujillo-Pisanty et al. 2011; Hernandez et al. 2012). Briefly: the six and seven parameter versions of the mountain model (Hernandez et al. 2010; Trujillo-Pisanty et al. 2011; Hernandez et al. 2012) were fit to the drug and vehicle datasets separately for each rat. Both models include parameters that specify the location and slope of the fitted surface along both the price and pulse-frequency axes as well as the maximal and minimal TA. The 7-parameter version includes an additional term that estimates the contribution of conditioned reward (Hernandez et al. 2010), thus accommodating the possibility that the lever and/ or the act of lever depression come to serve as secondary reinforcers. The fits were carried out using a MATLAB (the MathWorks, Natick, MA) procedure developed by Kent Conover, based on the non-linear least-squares routine in the optimization toolbox and resampling methods (Efron \& Tibshirani 1994). The data from each rat under the drug and vehicle conditions were resampled with replacement 1000 times by survey. The model was fit to each of the resulting datasets. Mean values for each parameter within condition were obtained by averaging the 1000 estimates. Confidence intervals were obtained by excluding the lowest and the highest 25 values of the 1000 estimates. This strategy allowed us to obtain unbiased estimates of the location parameters $\left(P_{e}\right.$ and $\left.F_{h m}\right)$ and their dispersions for each subject under each condition. Two different fitting strategies were used per model: in the location-specific approach, location parameters were estimated for each survey; the all-parameters-common approach estimated single location parameter values for an entire dataset (Hernandez et al. 2010; Trujillo-Pisanty et al. 2011; Hernandez et al. 2012). The Akaike information criterion (AIC) (Akaike 1974) was used to select the model and strategy that provided the best fit of the data. Difference vectors were then constructed by subtracting each of the 1000 parameter location-parameter estimates for the vehicle condition from the corresponding estimates for the drug condition. Confidence intervals were derived from the 2.5th and 97.5th percentiles of the difference vector. Differences were regarded as significant with an alpha level of 0.05 when zero fell outside the $95 \%$ confidence interval. This strategy allowed us to assess the effects of the drug within subject.

\section{Results}

Two rats were unable to meet the performance criteria and were excluded from the experiment. The six remaining rats learned to perform appropriately on frequency, price, and radial sweeps. Figure 2 shows the mean TA from a representative rat (subject Pharm10), for every trial in each type of sweep. Note that as expected, TA increased as a function of pulse frequency and decreased as a function of price. In other words, the stronger and cheaper the electrical reward, the more it 
was sought out by the rat and the less the rat engaged in alternate activities. In all sweeps, the upper asymptote is above 0.8 and the lower asymptote below 0.2 .

Histological analysis confirmed that the electrode tips were located within the MFB, at the level of the LH, in all the rats included in the experiment (Figure 3).

The fitting strategy allowed us to perform within-subject analysis and comparisons. An example is shown in Figure 4, which depicts the vehicle and drug data from subject 10, along with the corresponding surface fits and contour plots. The horizontal line with right-pointing triangular endpoints and the vertical line with diamond endpoints represent the estimated $F_{h m}$ and $P_{e}$ values, respectively; the surrounding shaded bands denote $95 \%$ confidence intervals. Note that the trajectory of the radial sweep passes close to $\left(P_{e}, F_{h m}\right)$, as intended. In all rats, the model fit the data well: adjusted $\mathrm{R}^{2}$ values ranged from $0.965-0.989$ in the vehicle condition and $0.961-0.988$ in the drug condition.

Figure 5 replots the data in Figure 4 so as to highlight any drug-induced shifts of the fitted 3D surface, The contour graph for the vehicle condition is shown twice, in the upper left as well as in the lower right quadrant. Note that Pimozide did not move the mountain along the frequency axis (as indicated by the horizontal lines with right-pointing triangular endpoints positioned at $F_{h m}$ ), whereas a clear $\left(-0.0857 \log _{10}\right.$ units) leftward shift along the price axis is evident (as indicated by the arrowhead between the projections of the vertical lines with diamond endpoints representing $P_{e}$ ). The bar graph summarizes the effect of the drug on the position of the mountain along the two axes.

Figure 6 summarizes the results from all subjects. Note that Pimozide failed to produce a statistically reliable change in the $F_{h m}$ parameter in any of the rats. In sharp contrast, the drug decreased $P_{e}$ in five of six rats. The mean shift along the price axis was $-0.0971 \log _{10}$ units (a 1.25fold decrease).

If the effect of Pimozide had varied systematically across test sessions as a result of tolerance or sensitization, this would have inflated the variance of the location-parameter estimates. Similar inflation of error variance would have been produced by systematic changes in baseline values as a result of the repeated drug administration. Figure 7 shows that the location-parameter estimates obtained in the vehicle and drug sessions did not vary systematically across test sessions. The values shown are expressed as within-session deviations from vehicle means and are averaged across subject.

\section{Discussion}

The importance of the $3 D$ perspective. A researcher studying ICSS by means of the conventional curve-shift method is, in effect, viewing the silhouette of the reward mountain in 2D while facing the pulse-frequency axis (Hernandez et al. 2010). When the silhouette shifts to the right, such an observer cannot be sure what movement of the mountain is responsible (Video 1, Figure 8). Although it is possible that the $3 \mathrm{D}$ structure indeed has moved rightward, it is also possible that the mountain has retreated along the price axis. Due to the diagonal orientation of the mountain surface, a displacement towards lower values along the price axis (hidden in the 2D view) drags the silhouette rightwards along the pulse-frequency axis. When we examine the results of the 
previous studies of Pimozide-induced changes in ICSS by means of the curve-shift method, we are confined to the 2D perspective of the observer described above. As a result, we cannot be sure whether the reported rightward displacement of rate-frequency curves reflects movement of the mountain along the pulse-frequency or price axes (Figure 8). In the present study, we eliminated this ambiguity by using a 3D measurement method that provides a view of the mountain from above, thus making the components of its displacement along the two axes readily apparent (Figure 5, Video 1). Whereas Pimozide produced no reliable movement along the pulse-frequency axis, the direction of the shifts reported in 2D studies (Gallistel \& Freyd 1987; Miliaressis et al. 1986; Rompré \& Wise 1989), the drug consistently displaced the reward mountain along the dimension that was hidden in the prior work: the axis representing reward cost.

Comparison to previous $2 D$ results. The reliable shifts revealed by our $3 \mathrm{D}$ method in 5 of 6 rats following administration of Pimozide at a dose of $0.1 \mathrm{mg} / \mathrm{kg}$ contrast with the results reported for doses in the $0.1-0.175 \mathrm{mg} / \mathrm{kg}$ range in $2 \mathrm{D}$ curve-shift studies. A $0.1 \mathrm{mg} / \mathrm{kg}$ dose failed to produce statistically reliable shifts in a study by Franklin (Franklin 1978), who used a runway to measure ICSS performance and the number of pulses in a fixed-frequency train as the strength variable. In a later runway study carried out by Stellar et al. (Stellar et al. 1983), $0.125 \mathrm{mg} / \mathrm{kg}$ produced a reliable shift along the pulse-frequency axis in only 1 of 6 rats. In studies carried out in operant conditioning chambers, the small leftward shifts in rate-frequency curves reported by Gallistel and Freyd (Gallistel \& Freyd 1987) failed to meet their criterion for statistical significance at a $0.1 \mathrm{mg} /$ $\mathrm{kg}$ dose, as did the shifts reported by Rompré and Wise (Rompré \& Wise 1989) at a $0.175 \mathrm{mg} / \mathrm{kg}$ dose. Doses in the $0.2-0.35 \mathrm{mg} / \mathrm{kg}$ range produced statistically significant leftward curve shifts in all the aforementioned studies.

Why were reliable shifts seen in the present $3 \mathrm{D}$ study but not in previous $2 \mathrm{D}$ work carried out with similar doses? We have demonstrated previously (Hernandez et al. 2010; Hernandez et al. 2012) that shifts along the price axis displace the silhouette of the mountain along the pulsefrequency axis, creating the illusion of movement along an axis orthogonal to the one along which the 3D structure is actually displaced. If so, why weren't shifts in the 2D silhouette seen in the prior work? Inspection of the contours in Figures 4 and 5 reveals that such shifts would have been very hard to detect in 2D studies. Note that the slope of the diagonal portion of the contour lines is shallow: the effect of a large change in price is offset by a modest change in pulse frequency. This follows from the fact that the intensity of BSR produced by MFB stimulation rises steeply as a function of pulse frequency (Leon \& Gallistel 1992; Simmons \& Gallistel 1994; Hernandez et al. 2010; Trujillo-Pisanty et al. 2011; Hernandez et al. 2012). Given that the shifts along the price axis reported here are only on the order of 0.1 common logarithmic units, the correspondingly smaller displacement of the silhouette of the mountain along the pulse-frequency axis would likely have fallen below the detection threshold in 2D studies. The 3D method offers greater sensitivity than the 2D method because shifts along either or both axes can be detected.

The benefits obtained from the 3D method do have a cost: three months were required to obtain a dataset from a single rat in the present study. Given the long period required for training and testing, obtaining dose-response information is very expensive. Regardless of the testing paradigm, Pimozide and other neuroleptics pose particular problems in this respect. Gallistel and Freyd (1987) did not discern a dependence of 2D curves shifts on the dose of Pimozide in the 
range of $0.2-0.6 \mathrm{mg} / \mathrm{kg}$. As the dose was raised (to $0.4 \mathrm{mg} / \mathrm{kg}$ and above), an increasing proportion of subjects ceased to perform. Rompré and Wise (1989) have argued that the attenuation and ultimate cessation of performance as the dose of neuroleptic is increased likely reflects depolarization inactivation of DA neurons due to summation between the excitatory influence of the electrical stimulation and the disinhibition of DA somata by blockade of autoreceptors. The attenuation of ICSS performance is exacerbated in our paradigm because of the requirement to pay much higher opportunity costs than those demanded in $2 \mathrm{D}$ curve-shift studies carried out in operant chambers. Typically the rat must only make a single lever press of infinitesimal duration to earn a reward in such 2D curve-shift studies. In contrast, the rats had to keep the lever depressed for substantial periods of time to earn a reward in the present study; the highest prices tested ranged from 18.9 - 38.1 s. Given the high reward costs and the likely onset of depolarization inactivation, it is not surprising that we could not obtain reliable performance with doses of 0.25 or above. The dose window for 3D tests is very narrow indeed in the case of this drug. In future work, 2D studies may play a role complementary to 3D studies by providing information about wider dose ranges, albeit with lower sensitivity and interpretative power.

Comparison to previous $3 D$ results. The results of the present study are highly consistent with those obtained by means of the 3D method with drugs that block the dopamine transporter. Cocaine and GBR-12909, displaced the mountain rightward along the price axis (Hernandez et al. 2010; Hernandez et al. 2012), whereas we show here that Pimozide displaces the mountain leftward along the same axis. Thus, the mountain moves in opposite directions in response to indirect agonists and to a receptor antagonist. Both Pimozide in the present work, and GBR-12909, the more specific of the DAT blockers employed in the previous work, failed to produce any statistically reliable shifts of the mountain along the pulse-frequency axis. In contrast, the tested doses of Pimozide and GBR-12909 produced reliable shifts along the price axis in 5/6 and 7/10 rats, respectively.

Interpretation of the shift along the price axis. The confinement of the shifts to the price axis has important implications in terms of the stage of processing at which DA neurotransmission contributes to BSR. In early work on the role of DA in ICSS, it was often proposed or implied that the rewarding effect arose from direct excitation of midbrain DA neurons that give rise to MFB projections (Crow 1972; Arbuthnott et al. 1970; Anlezark et al. 1974; Corbett \& Wise 1980; Fibiger et al. 1987). However, the axons of these neurons have high thresholds to activation by extracellular stimulation, and their excitability and conduction properties are poorly matched to the inferred properties of the directly stimulated substrate for self-stimulation of the MFB (Shizgal et al. 1980; Gallistel et al. 1981; Bielajew \& Shizgal 1982; Yeomans et al. 1988; Anderson et al. 1996; Shizgal 1997). Moreover, the behaviorally relevant direction of conduction in at least a portion of the directly stimulated neurons subserving the rewarding effect is rostro-caudal, opposite to the direction in which the midbrain DA neurons project to forebrain targets (Bielajew \& Shizgal 1986). To accommodate both the psychophysically derived portrait of the directly stimulated substrate and the ample evidence that DA neurons play an essential role in ICSS, the “descending-path" hypothesis was proposed (Bielajew \& Shizgal 1986; Shizgal 1997). This hypothesis states that the rewarding effect of MFB stimulation arises from the direct excitation of descending, myelinated projections of forebrain neurons that provide trans-synaptic input to 
midbrain DA neurons. Supporting evidence is provided by recent demonstrations that direct optical excitation of descending MFB inputs to the ventral tegmental area (VTA) supports operant responding. These inputs excite VTA DA neurons either directly (Kempadoo et al. 2013) or via disinhibition mediated by local GABAergic interneurons (Jennings et al. 2013).

Rate-frequency curves obtained via 2D measurements of ICSS have often been likened to dose-effect curves in pharmacology (Wise 1996; Bauco et al. 1993). This analogy would work well if direct activation of DA neurons were responsible for the rewarding effect of MFB stimulation. Just as the dose of a drug required to produce an effect of a given magnitude would be increased due to partial blockade of the receptors at which the drug acts, the "dose" (e.g., pulse frequency) of stimulation required to produce a given ICSS response rate would be increased by a DA receptor antagonist. However, this analogy may no longer hold if, as modern evidence suggests, the activation of the DA neurons is largely of trans-synaptic origin. Whether or not the analogy remains valid in the case of trans-synaptic activation depends on where the DA neurons are positioned in the depiction provided by Figure 1.

The key pivot point in Figure 1 is at the output of the integrator, the circuit or neural population responsible for spatio-temporal integration of the post-synaptic signals arising from the volley of stimulation-induced action potentials in the directly stimulated neurons. The analogy between ratefrequency and dose-effect curves would remain valid if the DA neurons functioned merely to relay signals from the directly stimulated neurons to the input of the integrator. In that case, a DA antagonist, such as Pimozide, would increase the pulse frequency required to drive integrator output to a given level. We have termed such an effect a change in the sensitivity of the BSR substrate, an effect strictly analogous to a change in the $K_{D}$ of a receptor or the $K_{m}$ of an enzyme. In early studies, the effect of DA agents on ICSS was attributed to such changes in sensitivity (Crow 1970; Esposito \& Kornetsky 1977), which are expressed in the mountain model as a change in $F_{h m}$ (Hernandez et al. 2010). The results of both our prior studies of the effects of indirect DA agonists (Hernandez et al. 2010; Hernandez et al. 2012) and the present work with a DA antagonist are inconsistent with such a view: these agents shift the mountain reliably along the price axis (i.e., they change $P_{e}$ ) while producing little or no change along the pulse-frequency axis (as indexed by $F_{h m}$ ). These consistent findings show that the DA agents act at or beyond the output of the integrator.

One way in which the action of a drug at or beyond the output of the integrator could alter ICSS is by rescaling that output (as represented by the right-facing triangle in Figure 1). We describe such an effect as a change in gain (Hernandez et al. 2010), an adjustment that alters the maximum payoff attainable without changing the pulse frequency required to reach this plateau. A change in gain is analogous to a change in the $V_{\max }$ of an enzymatically catalyzed reaction or a change in the receptor density. Changes in sensitivity and gain are independent (as are changes in $K_{m}$ and $\left.V_{\max }\right)$.

A model proposed by Moisan and Rompré (Moisan \& Rompré 1998) predicts that manipulations of DA neurotransmission will alter reward-system gain. According to their model, rewarding stimulation of the posterior mesencephalon activates midbrain DA neurons transsynaptically. Spatiotemporal integration of the effects of the stimulation-induced volley underlying 
the rewarding effect occurs either in the dendritic arbor of the DA neurons and/or in a neural network that feeds input to the DA neurons from the directly stimulated population. If so, indirect DA agonists and Pimozide would not change the pulse frequency required to produce a given level of firing in the DA neurons, which will have already been determined before the stimulationinduced signal reached the spike-generating region. In contrast, the post-synaptic impact of the induced level of firing would be altered by indirect DA agonists and Pimozide as they boost release or block post-synaptic receptors, respectively. The results obtained in the present study using Pimozide and in previous 3D studies using indirect DA agonists are consistent with such drug-induced changes in gain and thus with transposition of the Moisan and Rompré model to the MFB.

An alternative interpretation of the results follows from the treatment of payoff that the rewardmountain model has inherited from the generalized matching law (Baum \& Rachlin 1969; Rachlin 1971; Killeen 1972). In those accounts and in the reward-mountain model, payoff is computed by scalar combination of variables such as reward quality, reward probability, and reward costs. A property of scalar combination is that a change in the value of an input variable simply rescales the result of the calculation, increasing or decreasing it by a given proportion. Thus, a change in reward cost can have the same effect as a change in gain.

An influential view of the role of DA neurons in operant performance couches their contribution in terms of the proclivity to invest effort in pursuit of rewards (Salamone et al. 2003; Salamone et al. 2007; Salamone et al. 2009; Niv et al. 2006; Niv et al. 2007). This view is consistent with the notion that the effect of Pimozide reported here is related to subjective reward costs rather than to reward-system gain. For example, the drug may have altered the rats' perception and/or evaluation of the effort required to hold down the lever. Such an effect differs from the deficit in response initiation typically associated with neuroleptic action: it operates in a different plane. Delayed initiation would have depressed maximal time allocation, compressing the mountain vertically. However, the low dose of Pimozide employed here failed to do so: As shown in Table 1,TAmax values for the vehicle and drug conditions were reliably different in only 1 of 6 rats (Rat Pharm3). Rather than lowering the "altitude" of the mountain, the drug shifted its position horizontally along the price axis. By substituting manipulation of effort costs for the manipulation of opportunity costs used in the present study, it may prove possible, in future studies, to distinguish between the contribution to such changes in position due to alteration of reward-circuit gain and alteration of subjective perception and/or evaluation of the effort required to procure a reward.

Herrnstein's conceptualization of single-operant performance in terms of choice between pursuit of an experimenter-controlled reward and engagement in alternate activities (Herrnstein 1970; Herrnstein 1974) provides a third interpretation of the results. Given this view, the effect of Pimozide observed in the present study could be attributed to a drug-induced increase in the value of alternate activities. That said, we have argued elsewhere that a change in the value of alternate activities is unlikely to account for the large rightward shifts of the reward mountain along the price axis that are produced by continuous infusion of cocaine (Hernandez et al. 2010). 
Conclusions. By applying a 3D measurement method, we demonstrate consistent effects of Pimozide on ICSS at a dose below the detection threshold in earlier 2D studies and we resolve an ambiguity inherent in the 2D methods. Whereas the 2D methods cannot determine the stage of neural processing at which drugs alter ICSS, the 3D method reveals that Pimozide acts at or beyond the output of the circuitry that performs spatiotemporal integration of the neural signals arising from MFB stimulation. Previous studies employing indirect DA agonists (Hernandez et al. 2010; Hernandez et al. 2012) show that the effects of those drugs on MFB ICSS also arise from action at or beyond the output of the integrator. As expected, the direction of the effects produced by Pimozide and the indirect agonists are opposite. Positioning midbrain DA neurons at or beyond the output of the integrator is consistent with both psychophysical (Shizgal et al. 1980; Gallistel et al. 1981; Bielajew \& Shizgal 1982; Bielajew \& Shizgal 1986; Shizgal 1997) and optogenetic (Jennings et al. 2013; Kempadoo et al. 2013) findings.

The introduction of optogenetic methods (Yizhar et al. 2011) is rapidly providing new information about the structure of brain reward circuitry and the neurochemical identity of its components (Tsai et al. 2009; Witten et al. 2011; Jennings et al. 2013; Kempadoo et al. 2013). For these and other new tools to realize their full potential, behavioral methods with the appropriate sensitivity and specificity are required. The reward-mountain model and the associated 3D measurement method are very promising in this respect. Combining these with optogenetic interventions should help link effects of drugs on reward seeking to well-defined actions at particular points in an increasingly detailed and accurate map of the underlying neural circuitry.

\section{Figure captions.}

Fig 1 Graphical representation and summary of the mountain model. (A) In the initial stages of reward processing, the action potentials produced by direct electrical activation of MFB axons are integrated temporally and spatially by a neural circuit that transforms the effect of the stimulation-induce volley into a signal representing subjective reward intensity. The peak reward intensity by the stimulation is combined, in scalar fashion, with subjective estimates of the probability and cost of reward so as to compute the payoff from procurement of a stimulation train. The rat's allocation of time to pursuit of the rewarding stimulation depends on the computed payoff in relation to the value of competing activities (e.g., grooming, resting or exploring). (B) Time allocation is represented as a function of the strength (pulse frequency) and opportunity cost ("price") of the stimulation. Interventions acting prior to the output of the integrator shift the three dimensional (3D) structure along the pulse-frequency axis. Such shifts are measured as changes in the parameter of the reward-mountain model, $F_{h m}$, that locates the 3D structure along the pulse-frequency axis. (C) Interventions acting at or beyond the output of the integrator shift the 3D structure along the price axis, as measured by a change in the parameter of the reward-mountain model, $P_{e}$, that locates the structure along this axis. (D) Formal statement of the reward-mountain model. Modified from Trujillo-Pisanty et al.(2011).

Fig. 2 Time allocation data from subject Pharm10, under vehicle and Pimozide conditions. Data shown are means and 95\% confidence intervals. (A) During frequency sweeps, the pulse frequency varied across trials and the price was held constant. (B) During price sweeps, the price varied across trials, and the frequency was kept at a value that yielded maximal reward. 
(C) during radial sweeps, frequency and priced varied conjointly across trials. The same data are shown twice, once from the viewpoint of the pulse-frequency axis and once from the viewpoint of the price axis. In all cases the upper asymptote is above 0.8 , and the lower asymptote is below 0.2 .

Fig. 3 Electrode placement. The location of each electrode tip is shown. All placements fell within the boundaries of the MFB, at the level of the $\mathrm{LH}$, as determined using low magnification microscopy and the Paxinos and Watson atlas (2007).

Fig. 4 Mean time allocation, fitted surfaces, and contour maps. The data are from rat Pharm10. In the $3 \mathrm{D}$ graphs in the upper row, the fitted surfaces are shown in grey, frequency-sweep data are designated by pyramids, price-sweep data are designated by cubes, and radial-sweep data are designated by polyhedrons. Contour graphs of the fitted surfaces are shown in the lower row. In these graphs, values of the independent variables along frequency sweeps are designated by triangles, along price sweeps by squares, and along radial sweeps by circles. The values of $F_{h m}$, $P_{e}$ and are indicated by horizontal lines with right-facing triangular end points and vertical lines with diamond end points, respectively. The shaded regions surrounding the lines denote $95 \%$ confidence intervals.

Fig. 5 Contour-graph representation of the effect of Pimozide in rat Pharm10. The contour map for the vehicle condition is shown twice (upper left and lower right) to facilitate across-condition comparison of location-parameter values $\left(\log _{10} F_{h m}, \log _{10} P_{e}\right)$ along the frequency and price axes. The bar graph shows the size of the drug-induced change in the values of the location parameters. Pimozide failed to shift the reward mountain along the frequency axis but displaced the mountain leftward along the price axis.

Fig. 6 Pimozide-induced shifts for all subjects. Error bars denote $95 \%$ confidence intervals. In no case did the drug reliably alter $F_{h m}$, whereas it decreased $P_{e}$ reliably in 5/6 subjects.

Fig. 7 Normalized shifts in location-parameter values across sessions. Two surveys of the reward mountain were carried out in each session. For each rat, the two estimates of each location parameter for each session were averaged so as to produce a single estimate for that session. A set of difference scores for each rat was then obtained by subtracting the average locationparameter estimate obtained for that rat across the 6 vehicle sessions from the estimate for each individual session. This causes the values for the vehicle sessions to fluctuate around zero and expresses the values for the drug sessions as deviations from the vehicle baseline. The difference scores were then averaged across the 6 rats to obtain the plotted values (open symbols: vehicle; filled symbols: drug). The error bars represent the standard error of the mean. As expected from the within subject analysis (Figure 6), the $F_{h m}$ values for the drug and vehicle conditions overlap, whereas the $P_{e}$ values fall consistently below the vehicle baseline. These plots provide no indication of the systematic shifts over sessions that would be expected from tolerance or sensitization effects.

Fig. 8 Ambiguity of 2D curve shifts. They grey surface (a) represents the reward mountain in the baseline condition. Decreases in the sensitivity of the reward circuitry shift the mountain rightwards along the pulse-frequency axis (c), whereas decreases in reward-circuit gain, 
increases in subjective effort costs, or increases in the value of alternate activities (V(AA)) shift the mountain leftwards along the price axis (e). An observer views the silhouette of the reward mountain in 2D against a "screen" consisting of an opaque vertical plane (not shown) that cuts through the mountain at a particular price. The perspective of the observer is the same as that of an experimenter who employs the curve-shift method, and the silhouettes the observer sees $(b, d, f)$ are analogous to rate-frequency curves. Such an observer cannot determine whether the 3D structure has moved along the pulse-frequency $(c, d)$ or price $(e, f)$ axis. The same silhouette can be produced by displacement of the 3D structure along either of the two axes (f). (Adapted from (Hernandez et al. 2010).)

\section{Table caption}

Table 1 Estimates of maximal time allocation $\left(T A_{\max }\right)$ in the vehicle and drug conditions. The criterion for statistical reliability is whether or not zero falls within the $95 \%$ confidence interval surrounding the difference between the estimates for the vehicle and drug conditions. In five of six cases, administration of Pimozide $(0.1 \mathrm{mg} / \mathrm{kg})$ had no reliable effect on $T A_{\max }$.

\section{References}

Akaike, H., 1974. A new look at the statistical model identification. Automatic Control, IEEE Transactions on, 19(6), pp.716-723.

Anderson, R.M., Fatigati, M.D. \& Rompré, P.P., 1996. Estimates of the axonal refractory period of midbrain dopamine neurons: their relevance to brain stimulation reward. Brain research, 718(1-2), pp.83-88.

Anlezark, G.M. et al., 1974. Electrical self-stimulation in relation to cells of origin of catecholamine-containing neural systems ascending from the brain stem. The Journal of physiology, 237(2), pp.31P-32P.

Arbuthnott, G.W. et al., 1970. Behavioural effects of stimulation in the region of the substantia nigra. Journal of pharmacological sciences, 210, pp.61P-62P.

Arvanitogiannis, A. \& Shizgal, P., 2008. The reinforcement mountain: allocation of behavior as a function of the rate and intensity of rewarding brain stimulation. Behavioral Neuroscience, 122(5), pp.1126-1138. Available at: http://psycnet.apa.org/journals/bne/122/5/1126/.

Atalay, J. \& Wise, R.A., 1983. Time course of pimozide effects on brain stimulation reward. Pharmacology, biochemistry, and behavior, 18(4), pp.655-658.

Bauco, P., Wang, Y. \& Wise, R.A., 1993. Lack of sensitization or tolerance to the facilitating effect of ventral tegmental area morphine on lateral hypothalamic brain stimulation reward. Brain research, 617(2), pp.303-308.

Baum, W. \& Rachlin, H., 1969. Choice as time allocation. Journal of the Experimental Analysis of Behavior, 12(6), pp.861-874. 
Bielajew, C. \& Shizgal, P., 1982. Behaviorally derived measures of conduction velocity in the substrate for rewarding medial forebrain bundle stimulation. Brain research, 237(1), pp. $107-119$.

Bielajew, C. \& Shizgal, P., 1986. Evidence implicating descending fibers in self-stimulation of the medial forebrain bundle. The Journal of neuroscience : the official journal of the Society for Neuroscience, 6(4), pp.919-929.

Breton, Y.-A. et al., 2013. Validation and extension of the reward-mountain model. Frontiers in Behavioral Neuroscience, 7, p.125.

Breton, Y.-A., Marcus, J.C. \& Shizgal, P., 2009. Rattus Psychologicus: construction of preferences by self-stimulating rats. Behavioural Brain Research, 202(1), pp.77-91.

Corbett, D. \& Wise, R.A., 1980. Intracranial self-stimulation in relation to the ascending dopaminergic systems of the midbrain: a moveable electrode mapping study. Brain research, 185(1), pp.1-15.

Crow, T.J., 1972. A map of the rat mesencephalon for electrical self-stimulation. Brain research, 36(2), pp.265-273.

Crow, T.J., 1970. Enhancement by cocaine of intra-cranial self-stimulation in the rat. Life sciences, 9(7), pp.375-381.

Edmonds, D.E. \& Gallistel, C.R., 1974. Parametric analysis of brain stimulation reward in the rat: III. Effect of performance variables on the reward summation function. Journal of Comparative and Physiological Psychology, 87(5), pp.876-883.

Edmonds, D.E. \& Gallistel, C.R., 1977. Reward versus performance in self-stimulation: electrodespecific effects of alpha-methyl-p-tyrosine on reward in the rat. Journal of Comparative and Physiological Psychology, 91(5), pp.962-974.

Efron, B. \& Tibshirani, R.J., 1994. An Introduction to the Bootstrap 1st ed, Boca Raton: Chapman and Hall/CRC.

Esposito, R. \& Kornetsky, C., 1977. Morphine lowering of self-stimulation thresholds: lack of tolerance with long-term administration. Science (New York, NY), 195(4274), pp.189-191.

Fibiger, H.C. et al., 1987. The role of dopamine in intracranial self-stimulation of the ventral tegmental area. The Journal of neuroscience : the official journal of the Society for Neuroscience, 7(12), pp.3888-3896.

Franklin, K.B., 1978. Catecholamines and self-stimulation: reward and performances effects dissociated. Pharmacology, biochemistry, and behavior, 9(6), pp.813-820. 
Gallistel, C., Shizgal, P. \& Yeomans, J., 1981. A portrait of the substrate for self-stimulation. Psychol Rev, 88(3), pp.228-273.

Gallistel, C.R. \& Freyd, G., 1987. Quantitative determination of the effects of catecholaminergic agonists and antagonists on the rewarding efficacy of brain stimulation. Pharmacology, biochemistry, and behavior, 26(4), pp.731-741.

Gallistel, C.R. \& Leon, M., 1991. Measuring the subjective magnitude of brain stimulation reward by titration with rate of reward. Behavioral Neuroscience, 105(6), pp.913-925.

Gallistel, C.R. et al., 1991. Effect of current on the maximum possible reward. Behavioral Neuroscience, 105(6), pp.901-912.

Hernandez, G. et al., 2010. At what stage of neural processing does cocaine act to boost pursuit of rewards? PLoS ONE, 5(11).

Hernandez, G. et al., 2006. Prolonged rewarding stimulation of the rat medial forebrain bundle: neurochemical and behavioral consequences. Behavioral Neuroscience, 120(4), pp.888904.

Hernandez, G. et al., 2012. Role of dopamine tone in the pursuit of brain stimulation reward. The Journal of neuroscience : the official journal of the Society for Neuroscience, 32(32), pp. 11032-11041.

Herrnstein, R., 1974. Formal properties of the matching law. J Exp Anal Behav, 21(1), pp.159-164.

Herrnstein, R., 1970. On the law of effect. J Exp Anal Behav, 13(2), pp.243-266.

Heyman, G.M. \& Beer, B., 1987. A new approach for evaluating the behavioral effects of antipsychotic drugs. Trends in Pharmacological Sciences, 8(10), pp.388-393.

Jennings, J.H. et al., 2013. Distinct extended amygdala circuits for divergent motivational states. Nature, 496(7444), pp.224-228.

Kempadoo, K.A. et al., 2013. Hypothalamic Neurotensin Projections Promote Reward by Enhancing Glutamate Transmission in the VTA. Journal of Neuroscience, 33(18), pp.76187626.

Killeen, P., 1972. The matching law. Journal of the Experimental Analysis of Behavior, 17(3), pp. 489-495.

Leon, M. \& Gallistel, C.R., 1992. The function relating the subjective magnitude of brain stimulation reward to stimulation strength varies with site of stimulation. Behavioural Brain Research, 52(2), pp.183-193. 
Miliaressis, E. et al., 1986. The curve-shift paradigm in self-stimulation. Physiology \& behavior, 37(1), pp.85-91.

Moisan, J. \& Rompré, P.P., 1998. Electrophysiological evidence that a subset of midbrain dopamine neurons integrate the reward signal induced by electrical stimulation of the posterior mesencephalon. Brain research, 786(1-2), pp.143-152.

Niv, Y. et al., 2007. Tonic dopamine: opportunity costs and the control of response vigor. Psychopharmacology, 191(3), pp.507-520.

Niv, Y., Daw, N. \& Dayan, P., 2006. How fast to work: Response vigor, motivation and tonic dopamine. Advances in neural information processing systems, 18, p.1019.

Olds, J. \& Milner, P., 1954. Positive reinforcement produced by electrical stimulation of septal area and other regions of rat brain. Journal of Comparative and Physiological Psychology, 47(6), pp.419-427.

Paxinos, G. \& Watson, C., 2007. The rat brain in stereotaxic coordinates 6 ed, Elsevier/Academic Press.

Petry, N. \& Heyman, G., 1997. Rat toys, reinforcers, and response strength: An examination of the Re parameter in Herrnstein's .... Behavioural Processes, 39, pp.39-52.

Rachlin, H., 1971. On the tautology of the matching law. Journal of the Experimental Analysis of Behavior, 15(2), pp.249-251.

Rompré, P.P. \& Wise, R.A., 1989. Behavioral evidence for midbrain dopamine depolarization inactivation. Brain research, 477(1-2), pp.152-156.

Salamone, J.D. et al., 2009. Dopamine, Behavioral Economics, and Effort. Frontiers in Behavioral Neuroscience, 3, p.13.

Salamone, J.D. et al., 2007. Effort-related functions of nucleus accumbens dopamine and associated forebrain circuits. Psychopharmacology, 191(3), pp.461-482.

Salamone, J.D. et al., 2003. Nucleus accumbens dopamine and the regulation of effort in foodseeking behavior: implications for studies of natural motivation, psychiatry, and drug abuse. The Journal of pharmacology and experimental therapeutics, 305(1), pp.1-8.

Shizgal, P., 1997. Neural basis of utility estimation. Current Opinion in Neurobiology, 7(2), pp. 198-208.

Shizgal, P., 2012. Scarce means with alternative uses: Robbins' definition of economics and its extension to the behavioral and neurobiological study of animal decision making. Frontiers in Neuroscience, 6, p.20. 
Shizgal, P. et al., 1980. Behavioral methods for inferring anatomical linkage between rewarding brain stimulation sites. Journal of Comparative and Physiological Psychology, 94(2), pp. $227-237$.

Simmons, J.M. \& Gallistel, C.R., 1994. Saturation of subjective reward magnitude as a function of current and pulse frequency. Behavioral Neuroscience, 108(1), pp.151-160.

Sonnenschein, B., Conover, K. \& Shizgal, P., 2003. Growth of brain stimulation reward as a function of duration and stimulation strength. Behavioral Neuroscience, 117(5), pp.978994.

Stellar, J.R., Kelley, A.E. \& Corbett, D., 1983. Effects of peripheral and central dopamine blockade on lateral hypothalamic self-stimulation: evidence for both reward and motor deficits.

Pharmacology, biochemistry, and behavior, 18(3), pp.433-442.

Trujillo-Pisanty, I. et al., 2011. Cannabinoid receptor blockade reduces the opportunity cost at which rats maintain operant performance for rewarding brain stimulation. The Journal of neuroscience : the official journal of the Society for Neuroscience, 31(14), pp.5426-5435.

Tsai, H.-C. et al., 2009. Phasic firing in dopaminergic neurons is sufficient for behavioral conditioning. Science (New York, NY), 324(5930), pp.1080-1084.

Wise, R.A., 1996. Addictive drugs and brain stimulation reward. Annual review of neuroscience, 19, pp.319-340.

Witten, I.B. et al., 2011. Recombinase-Driver Rat Lines: Tools, Techniques, and Optogenetic Application to Dopamine-Mediated Reinforcement. Neuron, 72(5), pp.721-733.

Yeomans, J.S., Maidment, N.T. \& Bunney, B.S., 1988. Excitability properties of medial forebrain bundle axons of A9 and A10 dopamine cells. Brain research, 450(1-2), pp.86-93.

Yizhar, O. et al., 2011. Optogenetics in neural systems. Neuron, 71(1), pp.9-34. 


\section{Table 1}

\begin{tabular}{|c|c|c|c|}
\hline Rat & $\begin{array}{c}\boldsymbol{T A}_{\max } \\
\text { Vehicle }\end{array}$ & $\begin{array}{c}\boldsymbol{T A}_{\max } \\
\text { Drug }\end{array}$ & Significant \\
\hline Pharm2 & 0.949 & 0.948 & FALSE \\
\hline Pharm3 & 0.976 & 0.918 & TRUE \\
\hline Pharm6 & 0.999 & 1.000 & FALSE \\
\hline Pharm10 & 0.963 & 0.951 & FALSE \\
\hline Pharm11 & 0.832 & 0.823 & FALSE \\
\hline Pharm13 & 0.986 & 0.967 & FALSE \\
\hline
\end{tabular}


Figure 1.

$\begin{array}{cccccccc}\text { directly } & \text { aggregate } & \text { intensity- } & \text { intensity } & \text { peak } & \text { computation } & \text { behavioral } \\ \text { stimulated } & \text { spike } & \text { growth } & \text { allocation } \\ \text { neurons } & \text { rate } & \text { function } & \text { scaling detector } & & \text { of payoff } & \text { function }\end{array}$

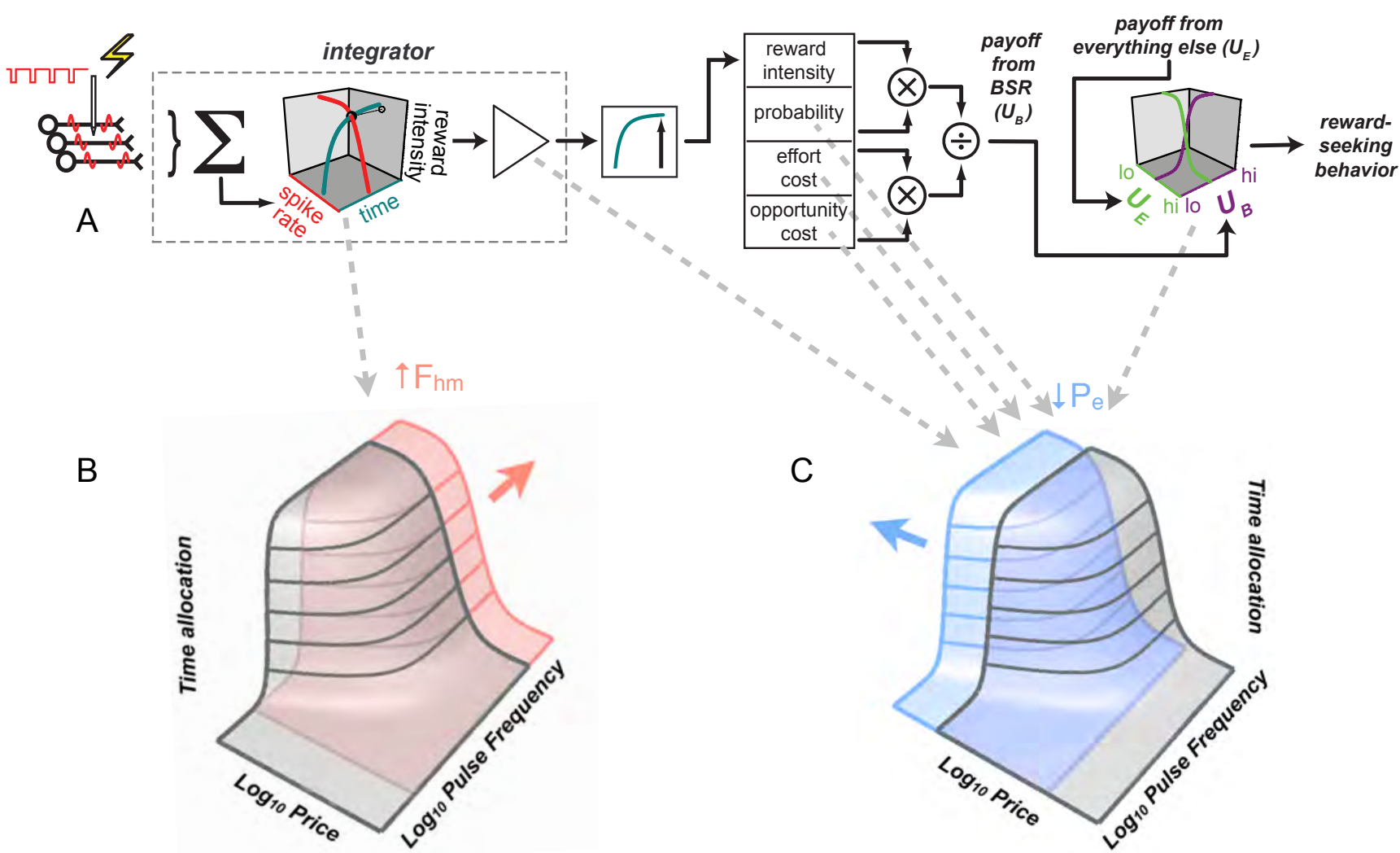

Mountain shifted along pulsefrequency axis

Mountain shifted along price axis

D

$$
\begin{aligned}
I & =I_{\max } \times \frac{f^{g}}{f^{g}+f_{h m}^{g}} \\
U_{B} & =\frac{I \times p}{(1+\xi) \times P} ; U_{E}=\frac{I_{\max } \times p}{(1+\xi) \times P_{E}} \\
T A & =\frac{U_{B}^{a}}{U_{B}^{a}+U_{E}^{a}} \\
\therefore T A & =\frac{\left(\frac{f^{g}}{f^{g}+f_{h m}^{g}}\right)^{a}}{\left(\frac{f^{g}}{f^{g}+f_{h m}^{g}}\right)^{a}+\left(\frac{P}{P_{E}}\right)^{a}}
\end{aligned}
$$

$a=$ price-sensitivity constant

$f=$ stimulation frequency

$f_{h m}=$ frequency producing a half-maximal reward

$g=$ reward-growth constant

$I=$ intensity of $B S R ; \quad I_{\max }=$ maximum intensity of $B S R$

$\xi=$ perceived exertion;

$p=$ reward probability; $P=$ price of a stimulation train

$P_{E}=$ price at which payoff from $I_{\max }=$ payoff from everything else $\left(U_{E}\right)$

$T A=$ time allocation;

$U_{B}=$ payoff from $B S R ; U_{E}=$ payoff from everything else 


\section{Frequency $(\mathrm{Hz})$}

A

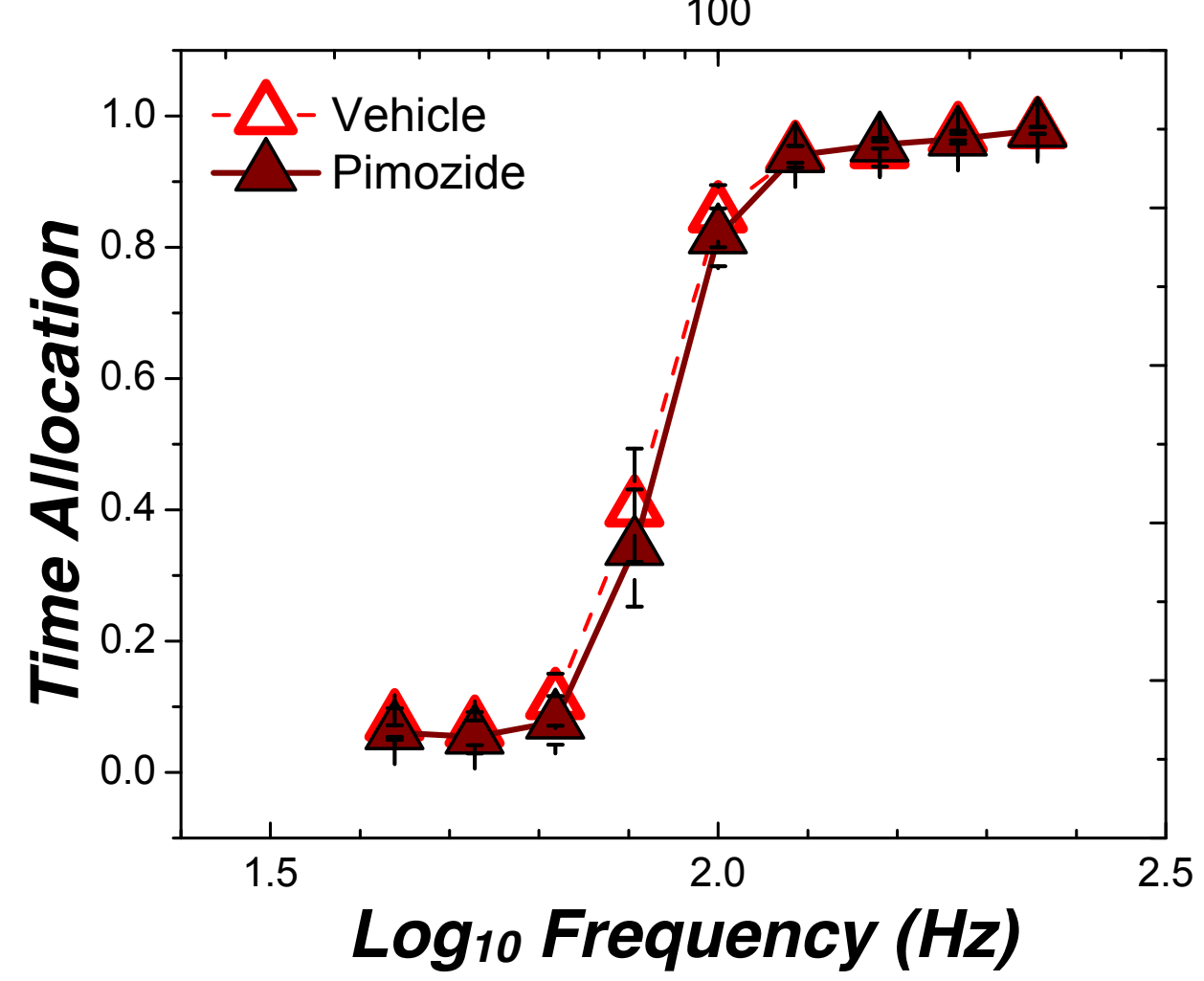

C

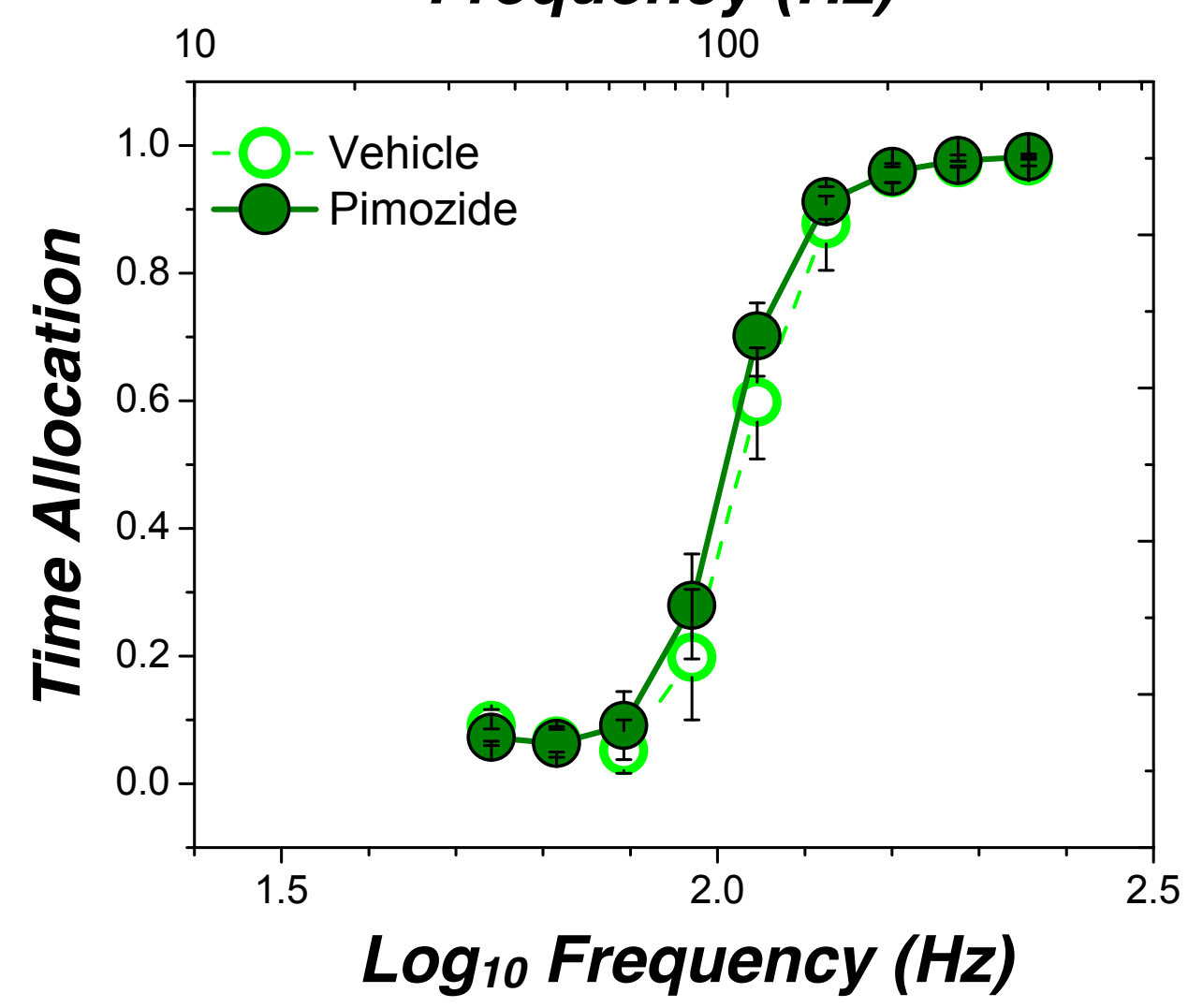

Price (s/reward)

B

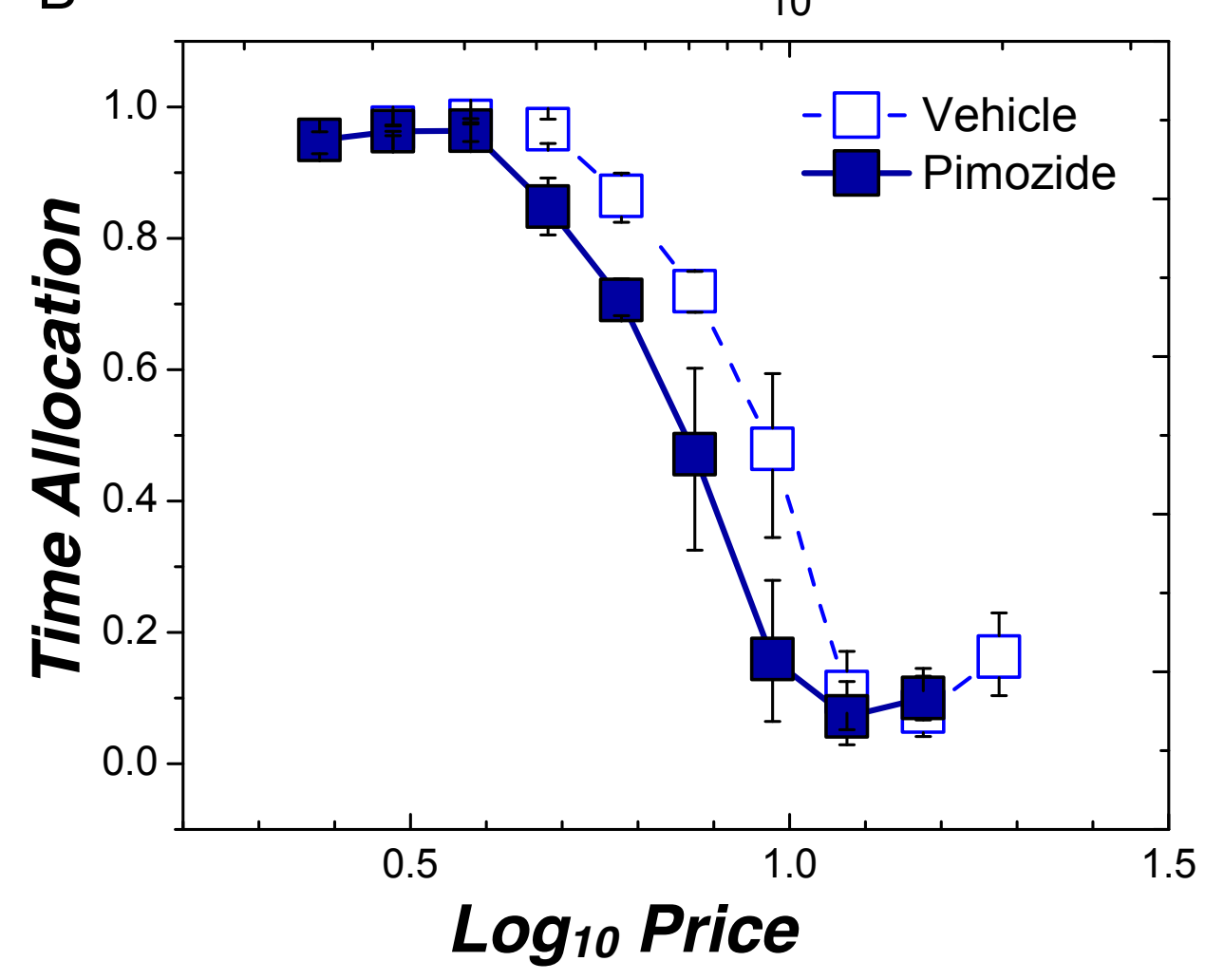

Price (s/reward)

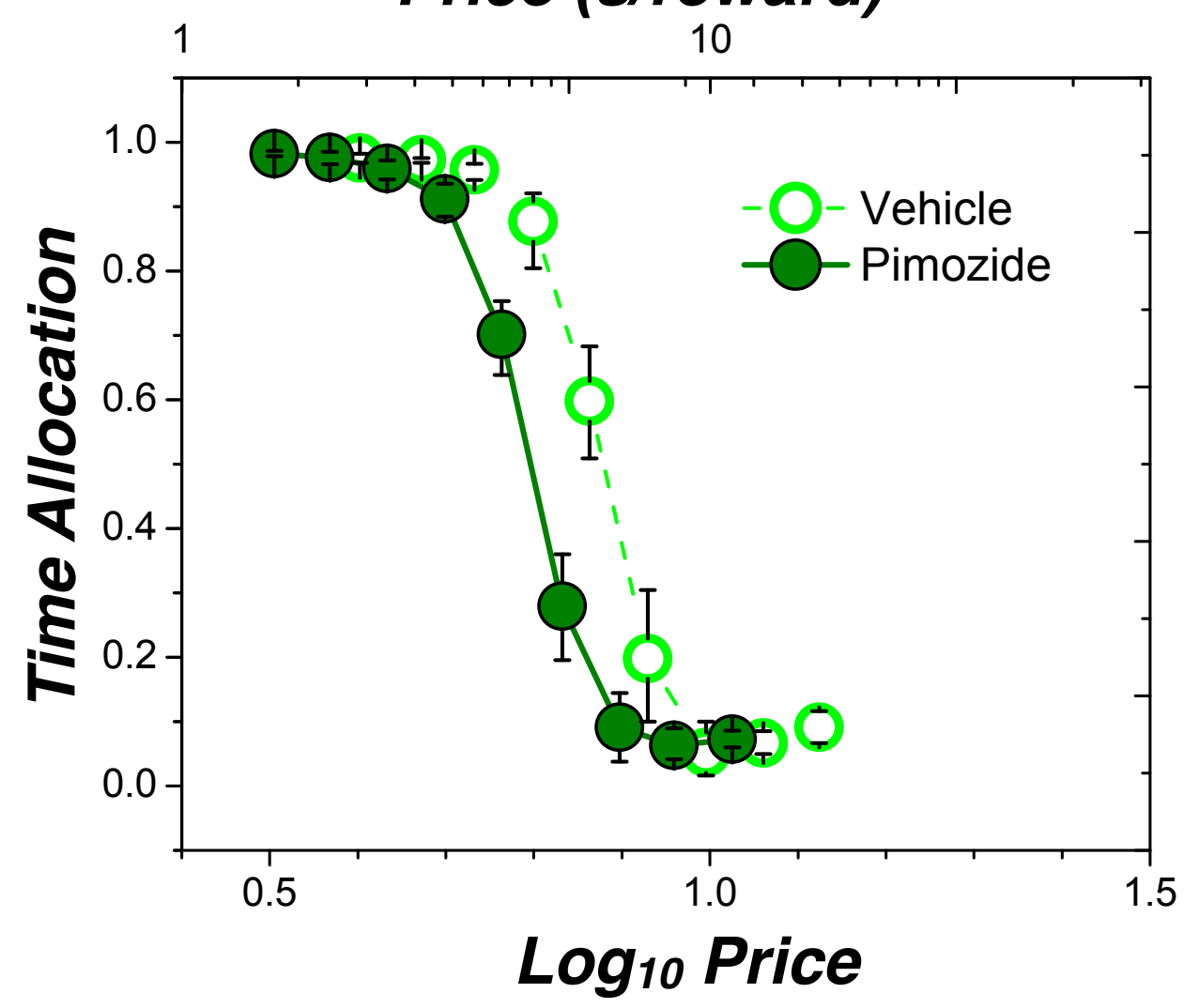




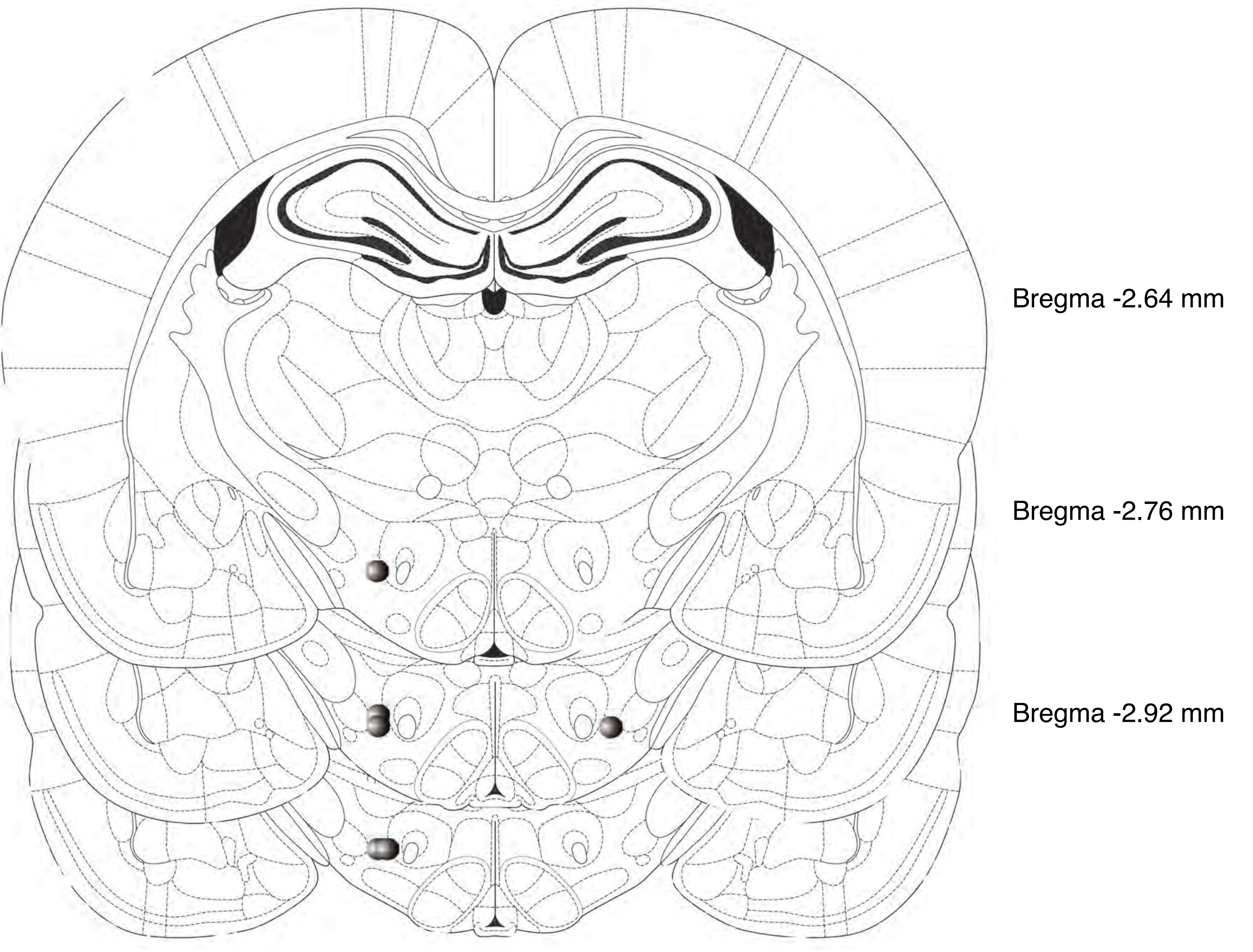

\section{Figure 3}



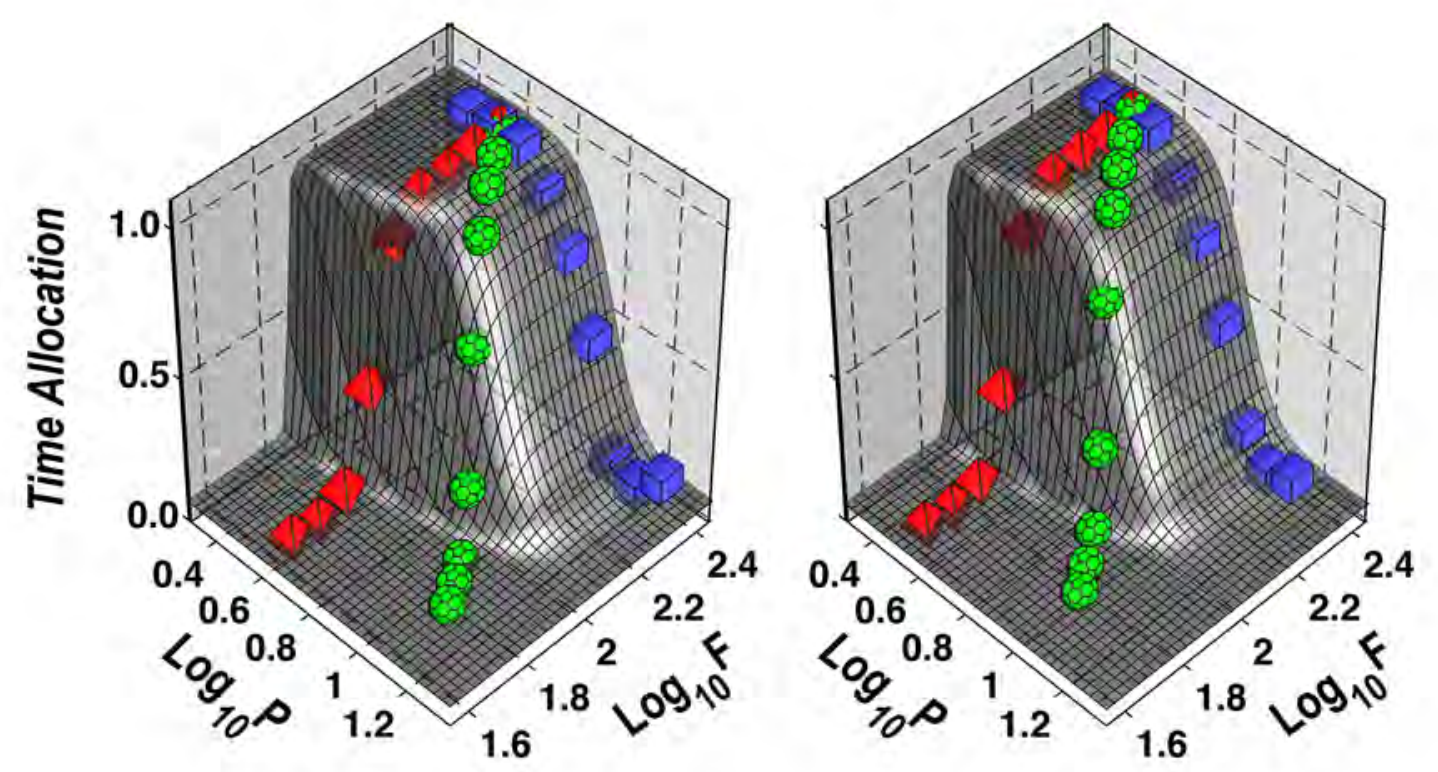

Price (s/reward)

Price (s/reward)
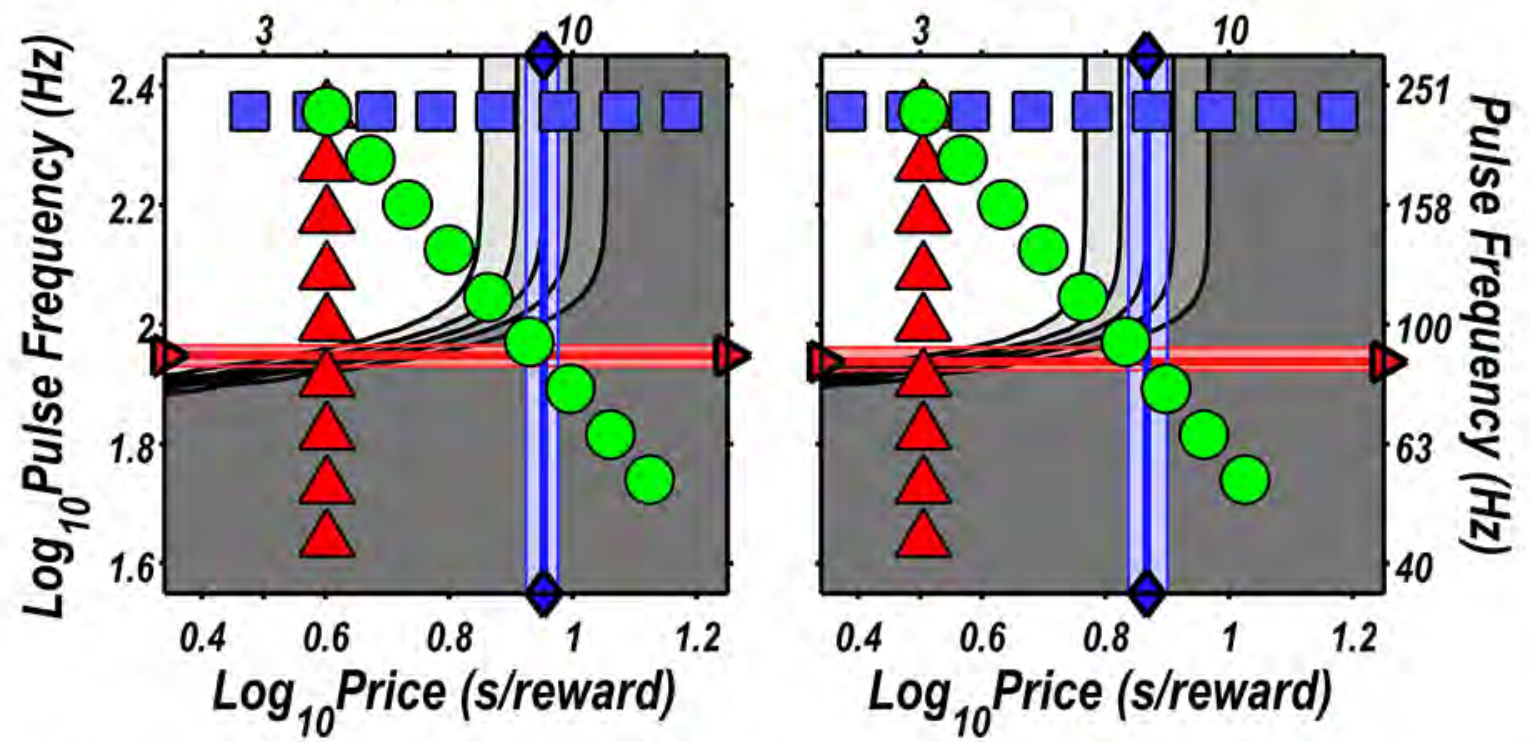

$\triangleright \log _{10} F_{h m}$

$\checkmark \log _{10} P_{\mathrm{e}}$

$\triangle$ Frequency

$\square$ Price

Radial

Time Allocation

0.1

0.2

0.3

0.4

0.5

0.6

0.7

0.8 


\section{Price (s/reward)}
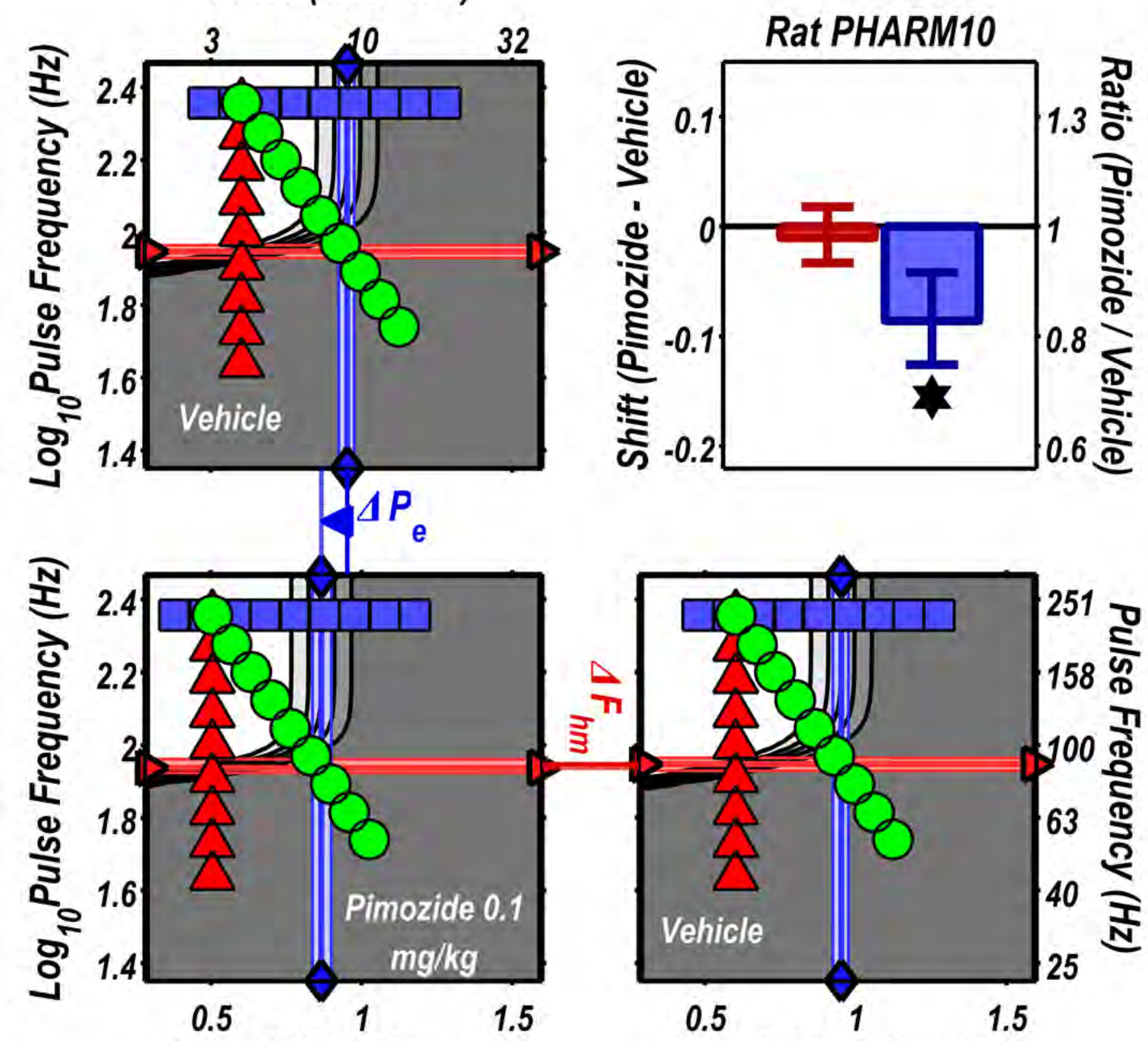

$\log _{10}$ Price (s/reward) $\quad \log _{10}$ Price (s/reward)

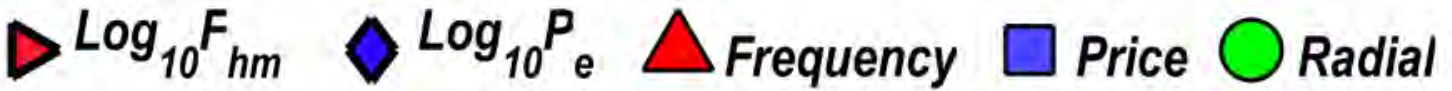
0.1
0.2
0.3
0.4
0.5
0.6
0.7
0.8

Time Allocation

Figure 5 


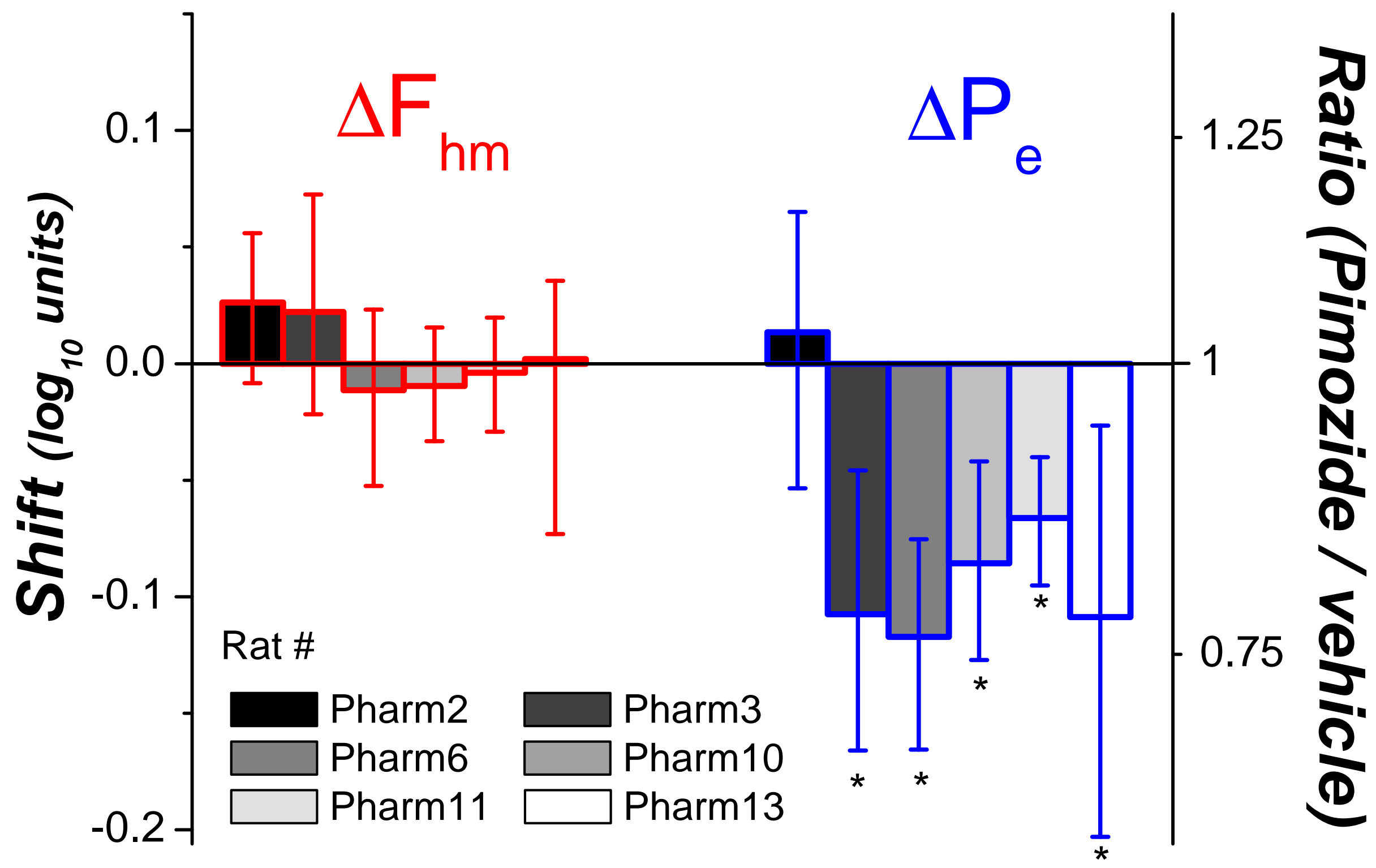




\section{D perspective}

2D perspective
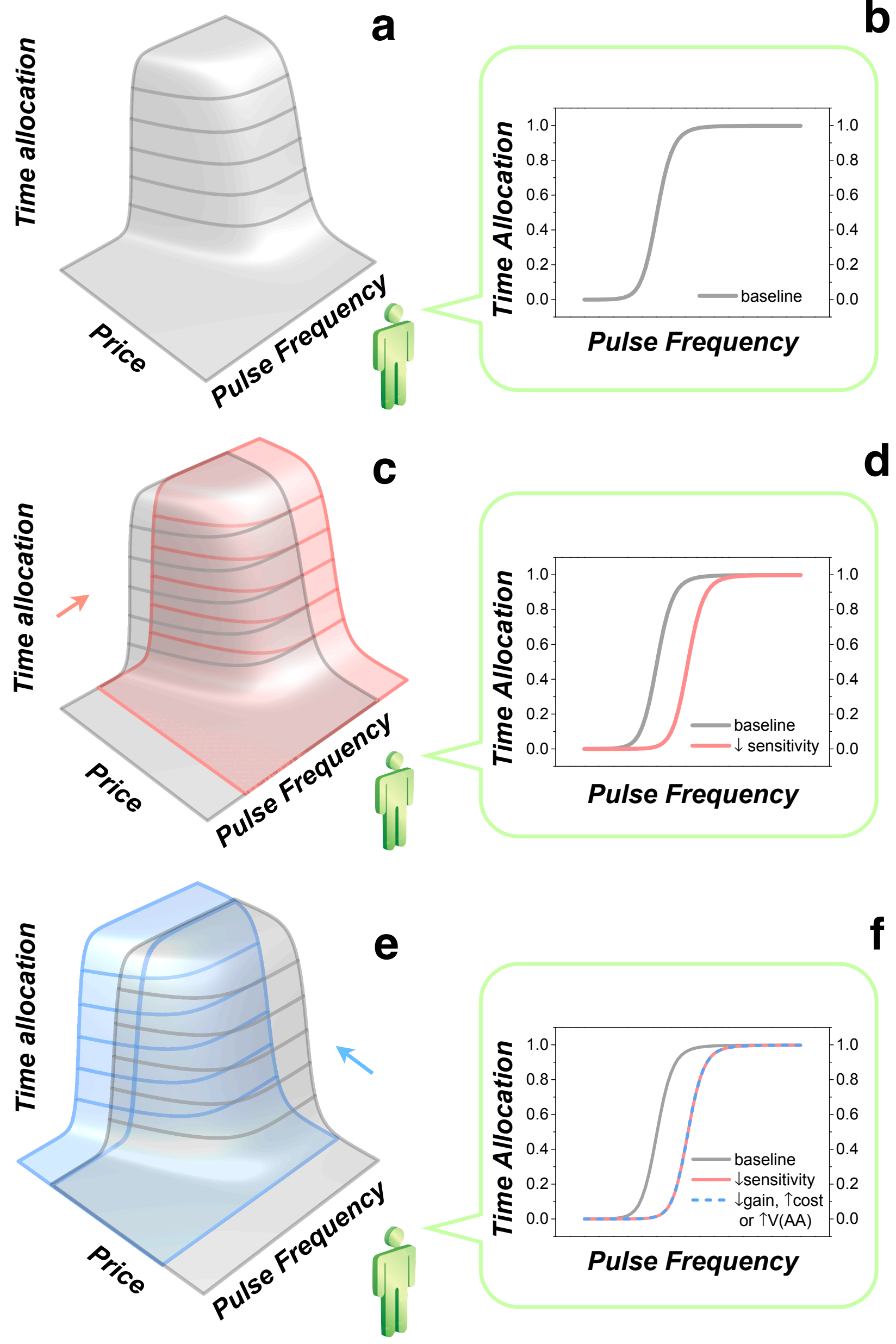

Figure 8 